\title{
Estresse, depressão e a relação com o "coping" em acadêmicos de medicina
}

\author{
Stress, depression and the relationship with coping in medical students
}

Estrés, depresión y la relación con la confrontación en estudiantes de medicina

Francisco Erinaldo Leite Pereira ${ }^{1}$, Charlene de Oliveira Pereira ${ }^{1}$, Yoshyara da Costa Anacleto Estrela1, Rafaella do Carmo Ribeiro ${ }^{1}$, Ariany Cibelle Costa Rezende ${ }^{1}$, Miguel Aguila Toledo', André Luiz Dantas Bezerra1, Larissa de Araújo Batista Suárez ${ }^{1}$, Joelly Holanda de Souza ${ }^{1}$, Milena Nunes Alves de Sousa ${ }^{1,2 *}$.

\section{RESUMO}

Objetivo: Analisar os problemas de estresse e depressão e sua relação com uso de coping em estudantes de Medicina. Métodos: Pesquisa de caráter transversal, quantitativa realizada com estudantes do curso de Medicina do Centro Universitário de Patos, Patos-PB. Para a coleta de dados utilizou-se três questionários validados no Brasil sobre coping, estresse e depressão. Os dados foram analisados a partir de estatísticas descritivas de frequência relativa e absoluta e de testes inferenciais de Correlações de Pearson. Resultados: A estratégia "confronto" se correlacionou diretamente com as fases de alerta e de resistência $(\rho=0,20 ; \rho=$ 0,$18 ; p<0,05$ ), a estratégia "fuga e esquiva" diretamente com a fase de resistência do estresse e a depressão $(\rho=0,20 ; \rho=0,27 ; p<0,05)$. Conclusão: A relação entre os problemas de saúde mental e a utilização de coping entre estudantes de Medicina foi importante para subsidiar reflexões, discussões e ações direcionadas para o cuidado dos alunos.

Palavras-chave: Estudantes de medicina, Saúde mental, Adaptação psicológica, Resiliência psicológica.

\begin{abstract}
Objective: To analyze the effects of the relationship between stress and depression problems in the use of coping of medical students. Methods: Cross-sectional, correlational, quantitative research conducted with students of the Centro Universitário de Patos, Patos-PB. For data collection were used three validated questionnaires in Brazil on coping, stress and depression. Data were analyzed from descriptive statistics of relative and absolute frequency and inferential tests of Pearson Correlations. Results: The "confrontation" strategy correlated positively with the alert and resistance phases $(\rho=0.20, \rho=0.18, p<0.05)$, the "escape and avoidance" strategy, positively with the stress resistance and depression $(\rho=0.20, \rho=0.27, p<0.05)$. Conclusion: The relationship between mental health problems and the use of coping among medical students was important to support reflections, discussions and actions aimed the care of students.
\end{abstract}

Keywords: Medical students, Mental health, Psychological adaptation, Psychological resilience.

\section{RESUMEN}

Objetivo: Analizar los problemas del estrés y la depresión y su relación con el uso de afrontamiento en estudiantes de medicina. Métodos: Investigación transversal y cuantitativa realizada con estudiantes de medicina de las Centro Universitário de Patos, Patos-PB. Para la recopilación de datos, se utilizaron tres cuestionarios validados en Brasil sobre la afrontamiento, el estrés y la depresión. Los datos se analizaron

1 Centro Universitário de Patos (UNIFIP), Patos - PB. * E-mail: minualsa@gmail.com

${ }^{2}$ Faculdade São Francisco da Paraíba (FASP), Cajazeiras - PB.

SUBMETIDO EM: 6/2020

ACEITO EM: 7/2020

PUBLICADO EM: $8 / 2020$

REAS/EJCH | Vol.Sup.n.55 | e4077 | DOI: https://doi.org/10.25248/reas.e4077.2020 Página 1 de 8 
sobre la base de estadísticas descriptivas de frecuencia relativa y absoluta y pruebas inferenciales de Correlaciones de Pearson. Resultados: La estrategia de "confrontación" se correlacionaba directamente con las fases de alerta y resistencia $(00,200 ; 0,18 ; p<0,05)$, la estrategia "escapar y evitar" directamente con la fase de resistencia a la tensión y la depresión $(0,200 ; 0,27 ; p<0,05)$. Conclusión: La relación entre los problemas de salud mental y el uso de la afrontamiento entre los estudiantes de medicina era importante para apoyar las reflexiones, discusiones y acciones dirigidas al cuidado de los estudiantes.

Palabras clave: Estudiantes de medicina, Salud mental, Adaptación psicológica, Resiliencia psicológica.

\section{INTRODUÇÃO}

Os cursos de ensino superior, principalmente os da área de saúde, são reconhecidamente causadores de estresse que comprometem a saúde mental dos indivíduos (PEREIRA GA, et al., 2015). O curso de Medicina é visto como um dos mais difíceis e trabalhosos, pois exige dedicação, esforço, sacrifício, resistência física e emocional dos estudantes. Ao ingressarem na faculdade, os acadêmicos têm sua rotina de vida alterada em diversos aspectos, as principais mudanças atingem o lazer e os relacionamentos interpessoais e podem acarretar desgaste emocional e estresse (NETO JAC, et al., 2013; PEREIRA GA, et al., 2015; CARDOSO JV, et al., 2019).

Vários estudos psicológicos, envolvendo os discentes do curso de medicina, têm sido realizados nos últimos anos. A formação médica, ainda hoje, permite ao indivíduo a possibilidade de anseios materiais e emocionais, de reconhecimento por seu intelectual e de satisfação pela carreira escolhida (ABOALSHAMAT $\mathrm{K}$, et al., 2015). No entanto, pesquisas têm demonstrado que o curso pode ser considerado potencialmente estressante e comprometer a qualidade de vida dos estudantes (PADOAVANI RC, et al., 2014; PEREIRA GA, et al., 2015).

Transtornos psicológicos da saúde, incluindo estresse e depressão, são comuns e bem documentados no mundo todo entre estudantes de Medicina (REZENDE ACC, et al., 2017; ESTRELA YCA, et al., 2018; CARDOSO JV, et al., 2019). Em comparação com a população em geral, os acadêmicos de Medicina têm uma prevalência maior de problemas de saúde mental. O status da saúde psicológica desses estudantes também se mostra com baixos níveis de autoeficácia e de satisfação com a vida (ABOALSHAMAT K, et al., 2015; CAMARGO VCV, et al., 2015).

São os primeiros anos da graduação médica, que se concentra a maior parte da incidência de sintomas depressivos e/ou de ansiedade, pois, diante das inúmeras novidades e dos desafios de sua formação, o estudante é demandado ao frequente uso e criação de recursos cognitivos e emocionais para o apoio da sua adaptação aos novos ambientes (PADOAVANI RC, et al., 2014; MOREIRA SNT, VASCONCELLOS RLSS e HEATH N, 2015).

A elevada prevalência de sintomas depressivos, ansiedade e estresse entre acadêmicos de Medicina têm efeitos danosos para a sua saúde e a realização pessoal. Baixo desempenho e queda na qualidade de vida são consequências com repercussões no âmbito familiar e institucional (PADOAVANI RC, et al., 2014; PEREIRA FEL, et al., 2017; YOSETAKE A, et al., 2019; SOUSA MNA, et al., 2020). Por conseguinte, no decorrer do estudo, a natureza dos estressores muda e os acadêmicos de Medicina podem experimentar um grande número de problemas emocionais e, provavelmente, precisam de mais suporte, o que induz à necessidade de estratégias de enfrentamento sejam mais eficazes nas distintas fases do desenvolvimento profissional (TARTAS M, et al., 2016; REZENDE ACC, et al., 2018; YOSETAKE A, et al., 2019).

Em âmbito internacional, as estratégias de enfrentamento são denominadas coping e tem sido entendido como o conjunto de estratégias cognitivas e comportamentais utilizadas pelos indivíduos para lidar com as demandas excessivas decorrentes de situações de estresse (VALLE TGM e MELCHIOR LE, 2010; OLIVEIRA $\mathrm{CT}$, et al., 2014). Na literatura internacional, estudos têm investigado o coping no ambiente universitário e suas relações com o estresse (GAN Y, et al., 2010; FAISAL-CURY A, et al., 2012; KOHSDDORF M, et al., 2015; SÁNCHEZ RT, et al., 2016). No Brasil, ainda há poucos estudos que investigam as estratégias de 
enfrentamento e suas consequências no âmbito acadêmico (BARDAGI MP e HUTZ CS, 2011). Apesar das extensas discussões na literatura mundial sobre os problemas de saúde mental nos acadêmicos de Medicina, não há estudos no cenário local que avaliem a realidade dos estudantes de Medicina e o impacto desses problemas na vida acadêmica.

A qualidade da saúde mental de estudantes universitários deve ser de interesse social e acadêmico, pois pode repercutir em suas vidas profissionais, afetar o cuidado para com o paciente ou levar os discentes ao abandono da profissão da área da saúde (ABOALSHAMAT K, et al., 2015; PEREIRA FEL, et al., 2017). Durante o período em que estão na universidade, as atividades acadêmicas, a elevada carga horária e as dificuldades em conciliar a vida acadêmica e a vida pessoal, com abdicação de momentos de lazer, interferem diretamente no equilíbrio emocional dos estudantes (BARDAGI MP e HUTZ CS, 2011; LOVEL GP, et al., 2015; YOSETAKE A, et al., 2019).

Estratégias de enfrentamento podem auxiliar no desenvolvimento de intervenções que promovem o bemestar psicológico e a qualidade de vida dos envolvidos (TARTAS M, et al., 2016; YOSETAKE A, et al., 2019). Desse modo, surgiu o interesse em realizar uma pesquisa, com a finalidade de responder as seguintes questões norteadoras: Quais as estratégias de enfrentamento adotadas pelo grupo diante de problemas de saúde mental? Qual a relação entre os problemas de saúde mental existentes em estudantes de Medicina e as das estratégias de enfrentamento dos mesmos?

Propõe-se, portanto, com esta pesquisa analisar a relação dos problemas de saúde mental (estresse e depressão) na utilização das estratégias de enfrentamento de estudantes de Medicina de uma faculdade do interior do Nordeste.

\section{MÉTODOS}

O Foi realizada pesquisa de caráter descritivo, transversal, com abordagem quantitativa, tendo como cenário de estudo a cidade de Patos, localizada no interior da Paraíba. $O$ estudo foi realizado no Centro Universitário de Patos (UNIFIP) com estudantes do curso de Medicina, do $1^{\circ}$ ao $5^{\circ}$ semestre (devido ter apenas tais períodos em execução no período da realização da pesquisa).

A amostra, não probabilística intencional determinada conforme critérios de inclusão e exclusão foi composta de 138 acadêmicos do curso de Medicina do UNIFIP. Como critérios de inclusão foram definidos: estudante regularmente matriculado no curso de Medicina e que demonstraram interesse em participar da pesquisa assinando o Termo de Consentimento Livre e Esclarecido (TCLE). Excluíram-se os estudantes que estavam fazendo qualquer tipo de tratamento psicológico durante o período de coleta de dados e não estavam blocados em nenhum período do curso.

Para coleta de dados foi utilizado os seguintes instrumentos: um questionário composto por questões objetivas com caracterização do perfil social, demográfico do estudante; o Inventário de Sintomas de Stress para Adultos de Lipp (ISSL) (LIPP MEN, 2000), para identificação dos sintomas de estresse; os sintomas depressivos foram investigados por meio do Inventário de Depressão de Beck (BDI) validado para população brasileira (GORENSTEIN C e ANDRADE LHSG, 1998), para as estratégias de Coping o Inventário de Estratégias de Coping (IEC) (LAZARUS RS e FOLKAMN S, 1984).

O questionário com variáveis sociodemográficas incluiu informações sobre idade, gênero, estado civil, lazer, bolsa de estudos para realizar o curso, satisfação com o curso e outras informações consideradas importantes. Essas informações auxiliaram na exploração de possíveis grupos de risco relacionados ao fenômeno estudado.

O ISSL apresenta um modelo quadrifásico do estresse, baseado no modelo trifásico de Selye e que identifica as fases de alerta, resistência, quase exaustão e exaustão (LIPP MEN, 2000). Neste modelo, a fase do alerta é tida como a fase positiva do estresse, na qual por meio da produção de adrenalina o ser humano se revitaliza, a sobrevivência é conservada e frequentemente uma sensação de plenitude é alcançada. $\mathrm{Na}$ fase de resistência, a pessoa, de modo, a manter sua homeostase interna, automaticamente tenta lidar com os estressores. Caso não consiga e os fatores estressantes persistirem, há quebra na resistência da pessoa 
e ela passa à fase de quase exaustão, onde começa o adoecimento de órgãos mais vulneráveis. A não remoção ou bloqueio dos fatores estressores ou uso do coping para aliviar o estresse, faz com que a pessoa entre na fase propriamente dita de exaustão e enfermidades podem ocorrer, como por exemplo: infarto, psoríase, úlceras entre outras (LIPP MEN, 2003).

Quanto ao BDI, o mesmo é uma escala sintomática de rastreamento de depressão, autoaplicada, composta por 21 itens que avaliam a sintomatologia depressiva presente na última semana (GORENSTEIN C e ANDRADE LHSG, 1998).

O inventário de estratégias de coping de Folkman e Lazarus validado no Brasil, "é um questionário que contém 66 itens que englobam pensamentos e ações que as pessoas utilizam para lidar com demandas internas ou externas de um evento estressante específico". O questionário avalia variáveis da personalidade e a escolha de estratégias em situações estressantes (SAVÓIA MG, et al., 1996) (Quadro 1).

Quadro 1 - Inventário de estratégias de coping segundo Folkman e Lazarus.

\begin{tabular}{|c|c|}
\hline Estratégia & Simplificado \\
\hline Autocontrole & $\begin{array}{l}\text { Refere-se ao empenho da pessoa em buscar o controle das emoções diante } \\
\text { dos estímulos estressantes }\end{array}$ \\
\hline Confronto & $\begin{array}{c}\text { São métodos nos quais o indivíduo apresenta uma conduta ativa em relação ao } \\
\text { estressor }\end{array}$ \\
\hline Afastamento & $\begin{array}{c}\text { Corresponde a estratégias de defesas, onde o ser evita confrontar-se com a } \\
\text { ameaça, não mudando a situação }\end{array}$ \\
\hline Suporte social & $\begin{array}{l}\text { Está relacionada ao apoio encontrado nos outros e no ambiente, sendo uma } \\
\text { ferramenta psicossocial positivo }\end{array}$ \\
\hline $\begin{array}{l}\text { Aceitação da } \\
\text { responsabilidade }\end{array}$ & $\begin{array}{c}\text { Nessa estratégia, o indivíduo aceita a realidade e envolve-se no processo de } \\
\text { lidar com o estresse }\end{array}$ \\
\hline Fuga e esquiva & $\begin{array}{l}\text { Consiste em fantasiar sobre soluções viáveis para as adversidades sem, no } \\
\text { entanto, tomar atitudes para de fato alterá-las. Pode ser descrita como os } \\
\text { esforços para escapar e/ou evitar a situação estressante }\end{array}$ \\
\hline $\begin{array}{l}\text { Resolução de } \\
\text { problemas }\end{array}$ & $\begin{array}{c}\text { Pressupõe o planejamento cabível para lidar com os fatores estressantes. Ao } \\
\text { invés de afastar ou anular a situação estressante de seu dia a dia, o indivíduo } \\
\text { opta por solucionar seu problema, mudar suas atitudes, sendo capaz de lidar } \\
\text { com as cobranças das pessoas e do ambiente ao seu redor, diminuindo ou } \\
\text { zerando a fonte geradora de estresse }\end{array}$ \\
\hline
\end{tabular}

Fonte: Pereira FEL, et al., 2019. Baseado em: Savóia MG, et al., 1996.

O IEC costuma ser utilizado para avaliar como os indivíduos lidam com as demandas internas ou externas de um evento estressante. É composto por 66 itens que incluem pensamentos e ações, e cuja intensidade é medida por meio de uma escala de 0 (não utiliza) a 3 pontos (utiliza em grande quantidade).

Os itens que compõem o inventário são divididos em oito fatores classificatórios, que foram reorganizados e mantidos, após a verificação da confiabilidade e validade à realidade brasileira (SAVÓIA MG, et al., 1996). São eles: confronto (itens 7, 17, 28, 34, 40, 47), afastamento (itens 6, 10, 13, 16, 21, 41, 44), autocontrole (itens $14,15,35,43,54$ ), suporte social (itens $8,18,22,31,42,45$ ), aceitação de responsabilidade (itens 9 , $25,29,48,51,52,62$ ), fuga e esquiva (itens 58,59 ), resolução de problemas (itens $1,26,46,49$ ) e reavaliação positiva (itens 20, 23, 30, 36, 38, 39, 56, 60, 63) (LAZARUS RS e FOLKMAN S, 1984).

A dinâmica da coleta de dados ocorreu por meio da entrega individual dos instrumentos da pesquisa no início da semana, sendo feito no horário de aula, e, na sequência, com o recolhimento ao final da mesma. Dessa forma, possibilitando tempo para analisar e responder as questões.

Os dados foram analisados através dos softwares Microsoft Excel®, versão 2007 e do software Statistical Package for the Social Sciences (SPSS), versão 21. Além de estatísticas descritivas de frequência relativa e absoluta e de testes inferenciais de Correlações de Pearson. Para todos os testes foi adotada uma significância estatística menor ou igual a 0,05, ou seja, $p<0,05$. 
A investigação é parte de um projeto guarda-chuva intitulado "Qualidade de Vida, Estresse, Síndrome de Burnout, Sintomas Depressivos e Coping em Estudantes de Medicina", o qual foi aprovado pelo Comitê de Ética das Faculdades Integradas de Patos, Número do Parecer: 1.582.097/2016.

\section{RESULTADOS E DISCUSSÃO}

O Gráfico 1 evidencia que a amostra foi composta por maioria do sexo feminino (58,7\%), com idade entre 17 a 24 anos $(83,3 \%)$, estafo conjugal solteiro $(96,4 \%)$, que moram com amigos ou colegas $(41,3 \%)$ e sem atividade remunerada $(95,7 \%)$.

Gráfico 1 - Descrição dos dados demográficos da amostra.

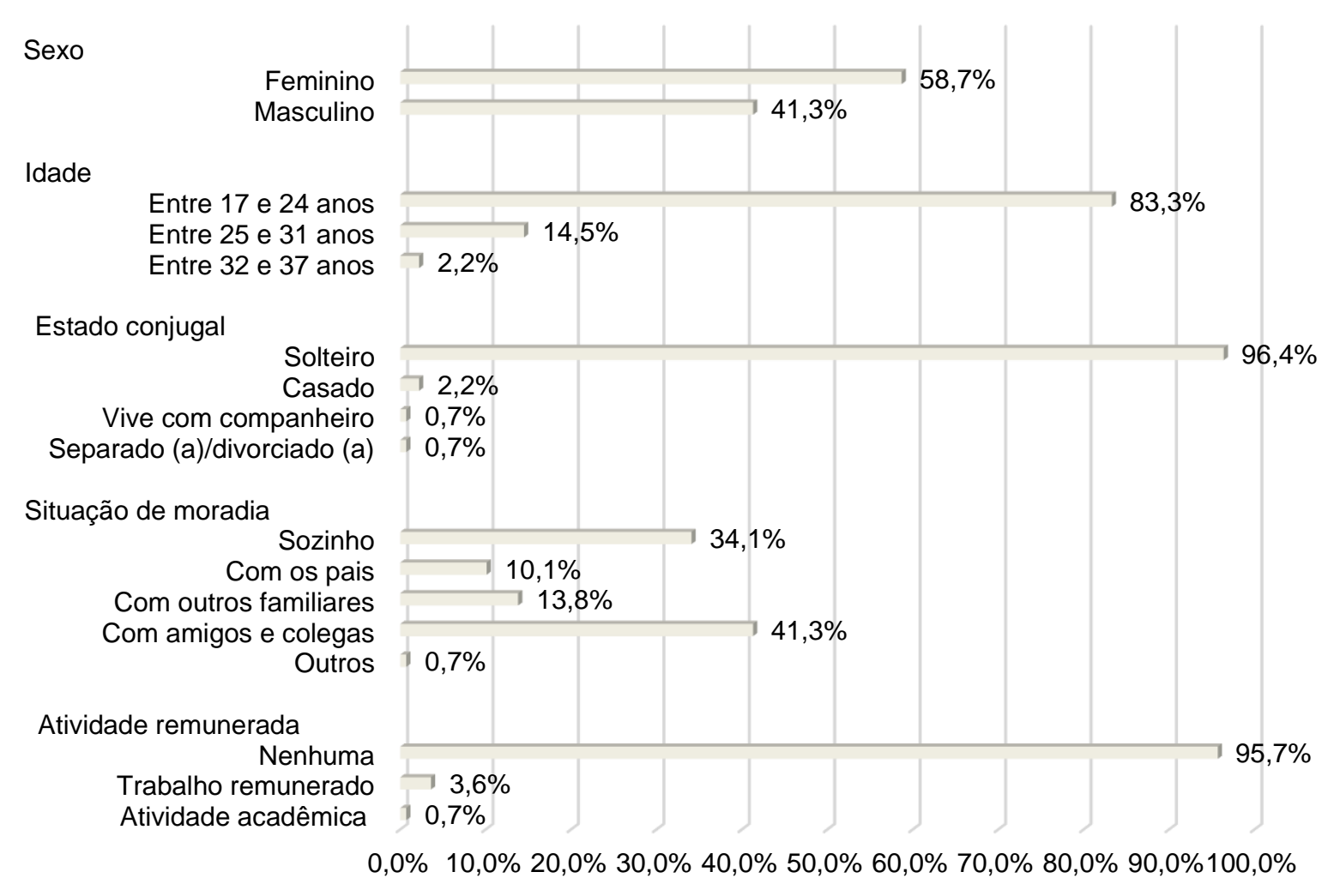

Fonte: Pereira FEL, et al., 2019.

A descrição dos dados relativos ao curso, identificada na Tabela 1, afirma que a amostra foi composta por estudantes dos cinco primeiros períodos do curso, com maior proporção no $5^{\circ}$ período $(31,9 \%)$ e os quais, na maioria, não possui bolsa $(81,9 \%)$.

Tabela 1 - Descrição dos dados relativos ao curso.

\begin{tabular}{|c|c|c|}
\hline Variáveis & $\mathbf{n}$ & $\%$ \\
\hline \multicolumn{3}{|l|}{ Período } \\
\hline $1 \stackrel{0}{\varrho}$ & 21 & 15,2 \\
\hline $2 \circ$ & 12 & 8,7 \\
\hline 30 & 33 & 23,9 \\
\hline 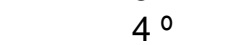 & 28 & 20,3 \\
\hline 50 & 44 & 31,9 \\
\hline \multicolumn{3}{|c|}{ Bolsa de estudos } \\
\hline Sim & 25 & 18,1 \\
\hline Não & 113 & 81,9 \\
\hline Total & 138 & 100 \\
\hline
\end{tabular}

Fonte: Pereira FEL, et al., 2019. 
Na sequência, a Tabela 2 mostra que a estratégia "confronto" se correlacionou positivamente com as fases de alerta, de resistência e com depressão. A estratégia 'aceitação da responsabilidade' se correlacionou positivamente com a fase de alerta e a estratégia 'fuga e esquiva' positivamente com a fase de resistência do estresse e a depressão. Estas correlações positivas e estatisticamente significativas indicam que existe uma relação diretamente proporcional, ou seja, à medida que uma aumenta a outra também pode aumentar.

Tabela 2 - Correlações entre estratégias de enfrentamento, estresse e depressão.

\begin{tabular}{ccccc}
\hline Variáveis & \multicolumn{3}{c}{ Estresse } & Depressão \\
\cline { 2 - 4 } & Fase de Alerta & $\begin{array}{c}\text { Fase de } \\
\text { Resistência }\end{array}$ & $\begin{array}{c}\text { Fase de } \\
\text { Exaustão }\end{array}$ & \\
\hline Confronto & $0,20^{*}$ & $0,18^{*}$ & 0,05 & $0,22^{\star *}$ \\
Afastamento & 0,11 & 0,14 & 0,02 & 0,08 \\
Autocontrole & 0,11 & 0,01 & $-0,03$ & 0,01 \\
Suporte social & 0,11 & 0,02 & 0,04 & $-0,05$ \\
Aceitação responsabilidade & $0,24^{\star *}$ & 0,08 & 0,07 & 0,11 \\
Fuga e esquiva & 0,04 & $0,20^{*}$ & 0,12 & $0,27^{* *}$ \\
Resolução de problemas & 0,07 & $-0,03$ & $-0,12$ & $-0,16$ \\
Reavaliação positiva & 0,06 & $-0,01$ & $-0,08$ & $-0,10$ \\
\hline
\end{tabular}

Legenda: ${ }^{*} p \leq 0,05 ;{ }^{* *} p \leq 0,01$.

Fonte: Pereira FEL, et al., 2019.

Os dados sociodemográficos coletados evidenciam que a maior parcela dos acadêmicos é de jovens, com faixa etária entre 17-24 anos, sendo 58,7\% identificada como sendo do sexo feminino. Caracterização convergente que enfatiza a presença feminina cada vez mais frequente nos cursos de Medicina, mudando assim o panorama até então encontrado no âmbito acadêmico, de forte presença do público Masculino (MATOS IB, et al., 2013; TORRES AR, et al., 2012).

Tal mudança pode ser observada ao longo das últimas décadas, e que recentemente vem se acentuando cada vez mais, em que registros foram verificados a partir dos profissionais formados em Medicina, conforme dados nacionais agrupados a partir de novos registros nos Conselhos Regionais de Medicina (CRM) a nível Nacional (SCHEFFER MC e CASSENOTE AJF, 2013; SMOLEN JR e ARAÚJO EM, 2017). Quanto à idade, estudos apontam que o fato de ser jovem e está na universidade é um fator de risco para o estresse, devido às novas responsabilidades que assumem (GOUVEIA MTO, et al., 2017; SANTOS FS, et al., 2017). Outros estudos também evidenciaram mais estresse em alunos jovens, sejam mestrandos, universitários ou prévestibulandos (FARIA RR, WEBER LND, TON CT, 2012; CARMARGO VCV, et al., 2015).

Diante disso, a Tabela 1 demonstra que a maioria dos alunos que responderam a pesquisas se encontram no $5^{\circ}$ período, assim é visto na literatura que o estresse, bem como, a sintomatologia depressiva em estudantes de Medicina, pode se modificar de acordo com o período no qual, o indivíduo se encontra, tendo em vista que o mesmo começa a se adaptar e a conseguir algumas estabilidades (ALVES JGB, et al., 2010). Para tanto, $81,9 \%$ da amostra não possuíam nenhum vínculo de bolsa de estudos que auxiliem no decorrer da vida acadêmica. Quanto ao fato de terem ou não bolsa, no início do curso é mais possível eventos estressores, por pressão de tempo, que questões financeiras entre outros (PRETO VS, et al., 2018).

Quanto às correlações entre estratégias de enfrentamento, estresse e depressão, os estudantes ao se encontrarem na fase de alerta se utilizam de estratégias de confronto $(\rho=0,20 ; p \leq 0,05)$ e aceitação e responsabilidade $(\rho=0,24 ; p \leq 0,05)$, corroborando acerca da saúde mental dos estudantes e a questão do estresse, verificaram em sua pesquisa que isso pode ocorrer nos primeiros períodos, no qual, advém de que no início do curso de Medicina pode-se ocorrer uma maior preocupação quanto o desenvolvimento acadêmico e futuro profissional, decorrente do novo formato de ensino e aprendizagem, o que não tende a ocorrer em alunos que se encontram em períodos mais avançado (VALLE TGM, e MELCHIOR LE, 2010).

Em contrapartida, ao se analisar a questão de confronto com depressão, foi observada correlação positiva $(\rho=0,22 ; p \leq 0,05)$. Isso tende a acontecer com os estudantes mais avançados, no qual poderiam estar mais 
exaustão ou quase exaustão e que já se poderia ser visto aspectos de adoecimentos, perda de concentração e sintomatologias depressivas (VALLE TGM e MELCHIOR LE, 2010). Diante disso, ressalta-se que o aumento de sintomas depressivos e estressores em universitários é assustador, pois, questões psicológicas podem acarretar não apenas problemas na saúde ou qualidade de vida do indivíduo, afetando, portanto, outros âmbitos de sua vida (VALLE TGM e MELCHIOR LE, 2010).

Ainda, a mesma amostra quando se encontra na fase de resistência responderam que utilizam estratégias de confronto $(\rho=0,18 ; p \leq 0,05)$; funga e esquiva $(\rho=0,20 ; p \leq 0,05)$. Essa última indica que $o$ indivíduo nessa fase tende a desenvolver estratégias de maneira fantasiosa, buscando encontrar soluções no qual saiam da situação ou as evitem (SAVÓIA MG, et al., 1996). Autores verificaram em sua pesquisa que os universitários na sua grande maioria, logo nos primeiros anos de faculdade são mais resistentes e conseguem se proteger de eventos estressores (VALLE TGM e MELCHIOR LE, 2010).

Outra pesquisa com grupo de adultos, em que foi verificado que $85 \%$ da amostra apresentam uma resistência, o que para o autor trata-se de algo preocupante, pois, fatores estressantes são frequentes e que muitas vezes quando não se tem controle diante do mesmo, pode-se levar o indivíduo a fase de exaustão ou quase-exaustão (DUTRA DL, et al., 2018). Quanto à questão de depressão com as estratégias de coping que os estudantes utilizam foi visto que, a mesma se correlacionou positivamente com a questão de fuga e esquiva. Os universitários, apesar de apresentarem uma resistência à questão de estresse, tendem a fugir e se esquivar de atividades.

Uma pesquisa realizada em Portugal com estudantes corrobora com os achados citados, indicando que $15 \%$ da amostra deles com sintomatologia depressiva tendem a adotar a estratégia de fuga e esquiva, entretanto, os mesmos ressaltam ainda que, isso ocorre ainda mais em indivíduos do sexo feminino que possuem uma renda baixa e são universitárias da área da saúde (ALVES JGB, et al., 2010; HIRSCH CD et al., 2015). Por fim, o ambiente acadêmico vem sendo apontado, em alguns estudos, como um causador de fenômenos que geram estresse e depressão em diversos níveis. Distintas situações específicas do dia-a-dia do estudante na faculdade/universidade tendem a acarretar tais sintomatologias (TORRES AR, et al., 2012; PADOVANI RC, et al., 2014; ABOALSHAMAT K, et al., 2015; SMOLEN JR e ARAÚJO EM, 2017; PRETO VS, et al., 2018).

Através da pesquisa pode-se perceber que os acadêmicos sabem lidar com situações adversas que venham a causar o estresse ou a depressão de forma significativa, e que para o seu sucesso como estratégia de enfrentamento a ajuda e o suporte dos familiares, de outros vínculos conquistados na faculdade são primordiais. Ao realizar a busca teórica para análise, houve certa dificuldade, uma vez que, as pesquisas científicas são moderadas, existindo poucos estudos que investiguem as relações de coping com estresse e depressão e suas consequências para os alunos de Medicina.

Entre as contribuições deste trabalho, pode-se dizer que é um estudo inovador e espera-se que esta pesquisa possa colaborar cientificamente para a promoção do estímulo ao uso das estratégias de enfrentamento em estudantes, promova reflexão, bem como, auxilie para o surgimento de discussões e debates que visem melhorar as condições de vida e a qualidade de vida dos acadêmicos. Um viés deste estudo é a falta de um feedback para os acadêmicos e a existência de estratégias que já se encontram na instituição, como o apoio psicológico aos estudantes.

\section{CONCLUSÃO}

Neste estudo foi possível observar que das estratégias de enfrentamento pesquisadas as que mais se destacaram foram os de confronto que mais se relacionou com as fases de alerta e resistência (do estresse) e, por conseguinte a depressão, aceitação da responsabilidade com a fase de alerta, e fuga e esquiva com a fase de resistência, tendo o maior índice na depressão. Com isso percebe-se que o acadêmico possui a necessidade de reconhecer o problema em si e tentá-lo solucionar antes que este problema afete sua qualidade de vida. Com a identificação por parte do estudante de Medicina sobre em qual fase do estresse se encontrava ou com quais sintomas depressivos apresentava, ajuda de certa forma a buscar formas para melhor lidar com a situação. 


\section{REFERÊNCIAS}

1. ABOALSHAMAT $\mathrm{K}$, et al. The impact of a self-development coaching programme on medical and dental students' psychological health and academic performance: a randomised controlled trial. BMC Medical Education, 2015; 15(1):1-13.

2. ALVES JGB, et al. Qualidade de vida em estudantes de Medicina no início e final do curso: avaliação pelo Whoqol-bref. Rev Bras Educ Med. 2010; 34(1), 91-6.

3. BARDAGI MP e HUTZ CS. Satisfação de vida, comprometimento com a carreira e exploração vocacional em estudantes universitários. Arq Bras Psicol. 2011;62(1),159-70.

4. CAMARGO VCV, et al. Estresse, depressão e percepção de suporte familiar em estudantes de educação profissionalizante. Estudos de Psicologia. 2015;32(4):595-604.

5. CARDOSO JV, et al. Estresse em estudantes universitários: uma abordagem epidemiológica. Revista de Enfermagem. 2019; 13(1):2-7.

6. DUTRA DL, et al. Avaliação da fase de estresse em estudantes da área da saúde. Estudos. 2018;45:21-15.

7. ESTRELA YCA, et al. Estresse e correlatos com características de saúde e sociodemográficas de estudantes de medicina. CES Med. 2018; 32(3): 215-225.

8. FAISAL-CURY A, et al. Coping style and Depressive Symptomatology during Pregnancyin a Private Setting Sample. The Spanish Journal of Psychology. 2012; 15(1): 295-305.

9. FARIA RR, et al. O estresse entre vestibulandos e suas relações com a família e a escolha profissional. Psicol. Argum.2012; 30(68):43-52.

10. FARIA RR, WEBER LND e TON CT. O estresse entre vestibulandos e suas relações com a família e a escolha profissional. Psicologia Argumento. 2012; 30(68): 43-52.

11. GAN Y, et al. Proactive and preventive coping in adjustment to college. Psychol Rec. 2010; 60(4): 643-658.

12. GORENSTEIN C e ANDRADE LHSG. Inventário de Depressão de Beck: propriedades psicométricas da versão em português. Rev Psiq Clin. 1998; 25(5): 245-50.

13. GOUVEIA MTO, et al. Quality of life and welfare of nursing university students: integrative review. Revista Enfermagem. 2017;6(3):72-8.

14. HIRSCH CD, et al. Coping strategies of nursing students for dealing with university stress. Rev. Bras. Enferm. (Online). 2015;68(5):783-90.

15. KOHLSDORF M. Coping strategies adopted by patients with chronic kidney disease inpreparation for transplant. Psychology, Community \& Health. 2015; 4(1): 27-38.

16. LAZARUS RS e FOLKMAN S. Stress, appraisal, and coping. New York, United States: Springer, 1984.

17. LIPP MEN. Manual do Inventário de Sintomas de Stress para Adultos de Lipp (ISSL). São Paulo: Casa do Psicólogo, 2000.

18. LIPP MEN. Mecanismos neuropsicológicos do stress: teoria e aplicações clínicas. São Paulo: Casa do Psicólogo, 2003.

19. LOVELL GP, et al. A cross-sectional investigation of depressive, anxiety, and stress symptoms and health-behavior participation in Australian university students. Nurs Health Sci. 2015;17(1):134-42.

20. MATOS IB, et al. Profissões e Ocupações de saúde e o Processo de Feminização: Tendências e Implicações. Athenea Digital. 2013;13(2):239-44.

21. MOREIRA SNT, VASCONCELLOS RLSS e HEATH N. Estresse na formação médica: como lidar com essa realidade? Revista Brasileira de Educação Médica. 2015; 39(4): 558-564.

22. NETO JAC, et al. Estudantes de Medicina sabem cuidar da própria saúde? HU Rev. 2013; 39(12): 45-53.

23. OLIVEIRA CT, et al. Adaptação Acadêmica e Coping em Estudantes Universitários Brasileiros: Uma Revisão de Literatura. Rev. Bras. Orientac. Prof. 2014; 15(2): 177-186.

24. PADOVANI RC, et al. Vulnerabilidade e bem - estar psicológicos do estudante universitário. Revista Brasileira de Terapias Cognitivas. Rio de Janeiro, RJ, v. 10, n. 1, p. 2-10, jun. 2014.

25. PEREIRA FEL, et al. Correlatos da qualidade de vida com características de saúde e demográficas de estudantes de medicina. Revista Brasileira de Qualidade de Vida. 2017; 9(3): 247-260.

26. PEREIRA GA, et al. Prevalência de síndromes funcionais em estudantes residentes de Medicina. Revista Brasileira de Educação Médica. 2015; 39(3):395-400.

27. PRETO VS, et al. Percepção de estresse nos acadêmicos de enfermagem. Revista Enferm UFPE. 2018;12(3):708-15.

28. REZENDE ACC, et al. Estratégias de coping utilizadas por acadêmicos de medicina. Revista Uningá. 2018; 55(3): 24-34.

29. REZENDE ACC, et al. Prevalência e sintomas depressivos em estudantes de Medicina/Saúde. Revista Coopex. 2017; 8(2):111.

30. SÁNCHEZ RT, et al. Intervenciones de enfermería para disminuir la sobrecarga en cuidadores: un estudio piloto. Rev Cuid. 2016; 7(1): 1171-84.

31. SANTOS FS, et al. Estresse em Estudantes de Cursos Preparatórios e de Graduação em Medicina. Rev. Bras. Educ. med. 2017; 41(2):194-200.

32. SAVÓIA MG, et al. Adaptação do inventário de estratégias de coping de Folkman e Lazarus para o português. Psic. USP. 1996; 7(1-2):183-201.

33. SCHEFFER MC e CASSENOTE AJF. A feminização da medicina no Brasil. Rev. bioét. 2013; 21(2): 268-77.

34. SMOLEN JR e ARAÚJO EM. Raça/cor da pele e transtornos mentais no Brasil: uma revisão sistemática. Ciências e Saúde coletiva. 2017; 22(12): 4021-30.

35. SOUSA MNA, et al. Correlatos de burnout com características de saúde e demográficas de estudantes de medicina. CES Med. 2020; 34(1): 27-39.

36. TARTAS M, et al. As estratégias de enfrentamento durante a educação médica preveem estilo de sucesso na carreira médica: um estudo longitudinal de 10 anos. BMC Med Educ 2016; 16: 186.

37. TORRES AR, et al. Inserção, renda e satisfação profissional de médicos formados pela Unesp. Rev. Bras. Educ. Méd. 2012; 36(1): 32-40.

38. VALLE TGM e MELCHIOR LE. ORGS. Saúde e desenvolvimento humano [online]. São Paulo (SP): Editora UNESP; São Paulo: Cultura Acadêmica, 2010. 257 p. ISBN 978-85-7983-119-5.

39. YOSETAKE A, et al. Estresse percebido em graduandos de enfermagem. Revista Eletrônica Saúde Mental Álcool Drog. (Online). 2019;14(2):117-24. 